\title{
Quick Detection System for Planck satellite
}

\author{
Juha Aatrokoski*, Anne Lähteenmäki, Merja Tornikoski \\ Metsähovi Radio Observatory, Finland \\ E-mail: jha@kurp.tkk.fi, alien@kurp.tkk.fi, Merja.Tornikoski@tkk.fi
}

\section{Esko Valtaoja}

Tuorla Observatory, Finland

E-Mail: Esko.Valtaoja@utu.fi

\begin{abstract}
Quick Detection System (QDS) is a software package designed to detect interesting point sources (for example, active galactic nuclei, AGNs) in the time-ordered datastream of the Planck satellite within approximately one week from the time of the observation. AGNs are rapidly variable, in the timescale of a few days to a few weeks, and any significant event must be investigated without delay.

This article presents the status of the software as an overview of the program features as well as a more detailed look at the data processing steps. This article is an adaptation of the poster which was presented at the Planck Consortium meeting in Ischia 17-20 April 2006.
\end{abstract}

$C M B$ and Physics of the Early Universe

20-22 April 2006

Ischia, Italy

\footnotetext{
* Speaker.
} 


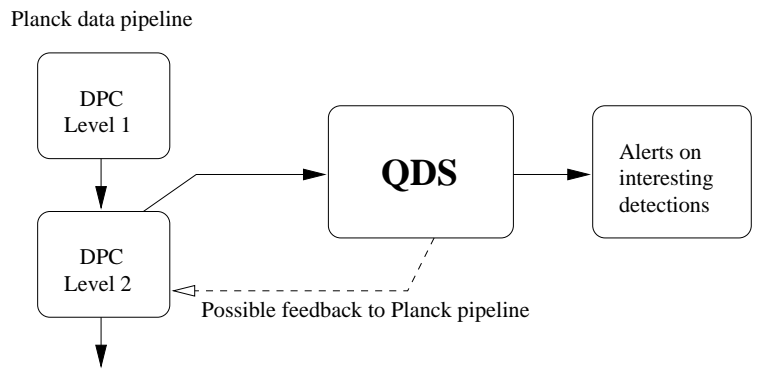

Figure 1: The place of QDS in the Planck data processing pipeline.

\section{General Features}

These are some of the general features of the software:

- Very configurable. Most configuration variables can have frequency- and detector-specific values as well as a default value.

- Support for arbitrary number of detectors and frequencies.

- Not strictly Planck-specific: the main restriction is that the data must be in similar rings as in Planck for ring normalization. This can be circumvented by using pre-normalized data.

- Can also be run in interactive mode where the results can be examined and source observation histories can be plotted as graphs.

- User manual (not yet complete).

\section{Processing Pipeline}

This section describes the processing pipeline (Figure 2 ) which is used to process the data rings from all the detectors.

The first step in the processing pipeline is the normalization of the data rings. The 60 very noisy sampling rings from the same sky circle are averaged together to produce a noise-reduced ring, the rings are adjusted to have a well-defined and consistent starting point and direction (Figure 3) and finally the sample units are converted from antenna temperature to flux density. Future work will implement caching of the normalized rings to reduce processing time on rings already processed once.

The normalized rings can be easily averaged with rings from other detectors in the same sky circle (detector grouping) and also with surrounding rings to further reduce noise.

The next step is filtering and thresholding, which are the actual steps that detect the point sources. Filtering uses a Mexican Hat Wavelet filter with the width parameter configurable for each detector either as a multiple of the beam FWHM or directly in arc minutes. Future plans for filtering include support for matched filters and optimal Mexican Hat width determination from the background power spectrum. 


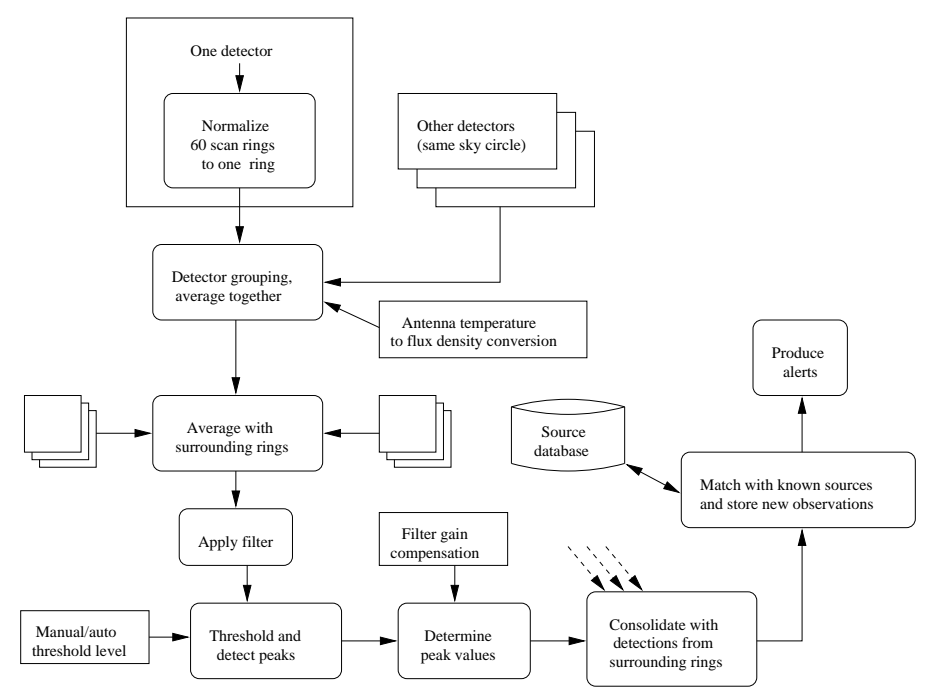

Figure 2: A detailed chart of the QDS processing pipeline. This illustrates how one ring from one detector group (and frequency) is processed. Multiple rings are processed in numerical order. Alerts produced by different detector groups are merged as the final step of the program run.

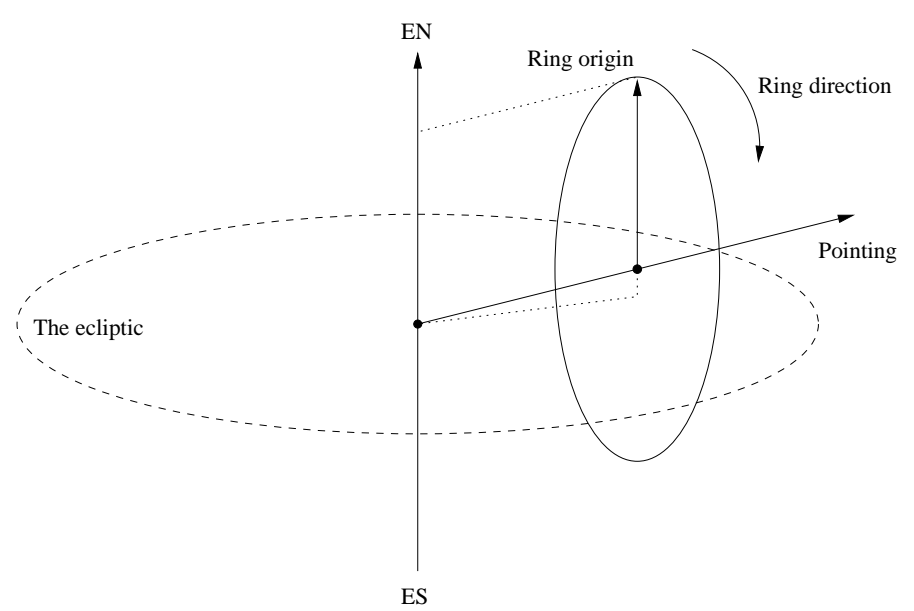

Figure 3: Ring normalization principles. A normalized ring starts from the ecliptic north and goes around the pointing vector in the clockwise direction.

After filtering the noise level $\sigma$ of the data is determined and the data is thresholded to detect point sources. The threshold is usually configured as a multiple of the noise level. Future plans for thresholding involve maybe using several thresholds for each ring, primarily depending on the galactic coordinates.

As a point source is usually visible in several rings, the detection results for each ring must be consolidated with the results from all the other rings being processed to remove duplicates and determine the correct values for coordinates and brightness. Finally those detections that are deemed interesting will produce an alert for the operator. 


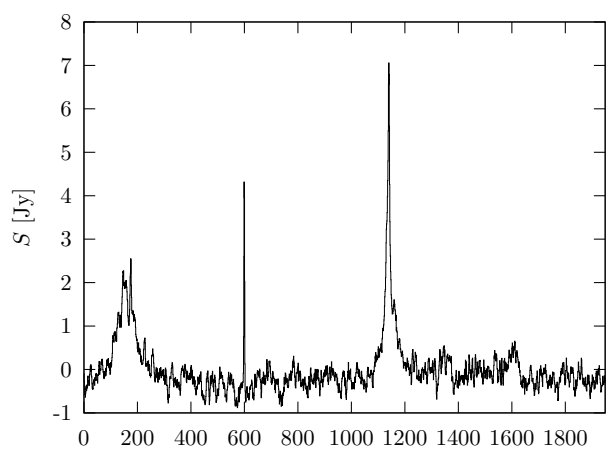

(a) Noise-reduced ring

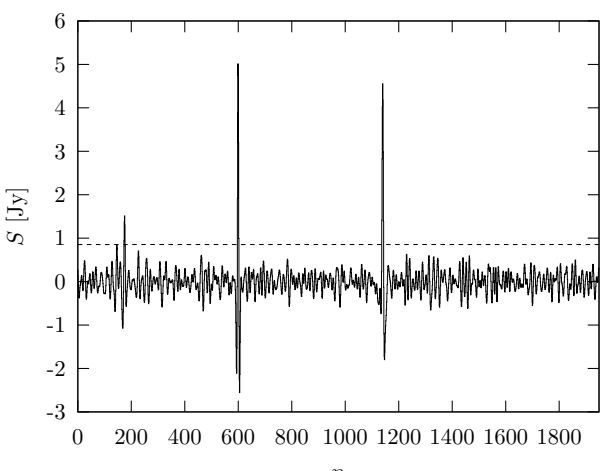

(b) Filtered ring

Figure 4: A ring after noise reduction and filtering. This example is from the $30 \mathrm{GHz}$ instruments. The unfiltered signal has been adjusted so that its mean is zero. The filter width used was the FWHM of the beam. A detection threshold of $4 \sigma$ is also plotted with dashes. Note the 5 Jy point source at $n=600$ and the large background structures at $n \approx 150$ and $n \approx 1150$ in the noise-reduced ring, and the detected point source and the spurious detections at the corresponding locations in the filtered ring.

\section{Alert Criteria}

A detection must satisfy one of the following criteria to be deemed interesting and produce an alert:

- The source is brighter than ever (by a certain factor):

$$
S>c S_{\max }
$$

- The source is brighter than the previous, recent enough observation of it:

$$
S>c S_{\text {prev }} \wedge t-t_{\text {prev }}<T
$$

- The source has an inverted spectrum.

- The source is previously unknown i.e. it is not in the point source database used by QDS. Most of these will probably be spurious due to noise and other artifacts in the signal.

\section{Further Information}

Further information about Planck science in Metsähovi and Tuorla can be found at the address http://kurp.tkk.fi/quasar/planck/index-en.htm Questions specifically about QDS can be sent to gds@kurp.tkk.fi. 